\title{
Emotional impact of genetic trials in progressive paediatric disorders: a dose-ranging exon-skipping trial in Duchenne muscular dystrophy
}

\author{
M. E. Garralda, ${ }^{\star}$ H. McConachie, $\$$ A. Le Couteur,$\S$ S. Sriranjan, $\dagger$ I. Chakrabarti, 9 S. Cirak, $\neq$ \\ M. Guglieri, ${ }^{\star *}$ K. Bushby ${ }^{\star *}$ and F. Muntoni $\neq$ \\ ${ }^{*}$ Academic Unit of Child and Adolescent Psychiatry, Imperial College London \\ $\dagger$ GOSH Rotation \\ \$Dubowitz Neuromuscular Centre, UCL Institute of Child Health, London \\ \$Institute of Health and Society, Newcastle University \\ INorthern Deanery, and \\ ${ }^{*}$ Institute of Genetic Medicine, International Centre for Life, Newcastle University, Newcastle, UK
}

Accepted for publication 18 March 2012

\author{
Keywords \\ clinical trials \\ methodology/study \\ design, gene therapy, \\ neuromuscular disease, \\ paediatric \\ Correspondence: \\ Elena Garralda, Academic \\ Unit of Child and \\ Adolescent Psychiatry, \\ Imperial College London \\ St Mary's Campus, \\ Norfolk Place, London \\ W2 1PG, UK \\ E-mail: \\ e.garralda@imperial.ac.uk
}

\begin{abstract}
Background Gene-modifying trials offer hope for improvement in chronic paediatric disorders, but they may also lead to disappointment and have an adverse emotional effect on families. This study aimed to examine emotional impact on participants in a paediatric exon-skipping trial.

Methods Nineteen male children with Duchenne muscular dystrophy (DMD), and their parents, taking part in a dose-ranging study of an i.v. administered morpholino splice-switching oligomer (which can restore the reading frame in DMD and induce dystrophin expression) underwent a psychosocial/psychiatric examination at trial entry. Emotional impact was assessed at trial completion using questionnaires.

Results The mean child age was 8.9 years (SD 2.1); 13(68\%) were attending mainstream school. Most families were well adjusted psychosocially at trial entry. Post-trial median child emotional impact scores were $5 / 10(n=18)$, but impact was rated as positive by $6 / 14(42 \%)$, neutral/mixed by $5(35 \%)$ and negative by $3(21 \%)$. Median post-trial psychosocial/psychiatric change scores in children and parents were minimal. Actual post-trial negative impact was statistically significantly associated with higher expected impact at trial entry, at which time the families of the three children displaying actual negative impact reported higher family stress levels in combination with a variety of other psychosocial risks factors.

Conclusions In carefully selected families with low levels of psychosocial stress/distress at trial entry, and with good support from paediatric research units (including psychiatric input when required), genetic trials in progressive disorders such as DMD can have a predominantly positive or neutral emotional impact. Nevertheless, negative impact is reported by a minority of families and possible psychosocial predictors deserving further scrutiny have been identified.
\end{abstract}

\section{Introduction}

The recent advent of genetic therapies is starting to bring new hope to children with progressive disorders such as Duchenne muscular dystrophy (DMD). These therapies are, however, still at early stages in clinical trials: the expected clinical implications are minimal or uncertain, and there are a number of reasons why they could have a de-stabilizing effect on the family's 
adaptation to the illness. First, the trial will disrupt usual routines and bring into sharper focus the deteriorating condition in the child, possibly re-awakening underlying concerns. High levels of mental distress have been noted in mothers of children with DMD at the stage when knee-ankle-foot orthoses are introduced to prolong independent motility, which has been attributed in part to having to confront the reality of the child's deterioration (Garralda et al.2006). Second, trials may generate unwarranted hope - as in 'therapeutic misconception' whereby in spite of full informed consent patients can still overestimate the beneficial effects of the therapeutic intervention and underestimate its adverse side effects (Kimmelman \& Palmour 2005; Henderson et al. 2006) - and this may lead to subsequent disappointment and distress. Lastly, the trial will make demands on the family in relation to time, travel and hospital contact: this may prove especially stressful for families at the stage of adapting to deteriorating mobility in their child.

However, even though research ethics committees often express concerns about protecting children they see as specially 'vulnerable' (Angell et al. 2010), little is known about the psychological effect that taking part in research trials generally has on children and their families/parents, nor on the specific effects of participating in genetic trials.

A previous proof-of-concept genetic trial exploration in children with DMD identified therapeutic misconceptions in $1 / 8$ families and an emotional impact from the trial in $2 / 5$ children, both associated with high levels of psychosocial/psychiatric stress at trial entry (Garralda et al. 2011).

The main aims of the present study were to explore the possible emotional impact on children with DMD and their parents of taking part in an exon-skipping clinical trial to evaluate the drug safety profile and to identify psychosocial/psychiatric predictors of emotional impact. It was hypothesized that a minority of children would experience an adverse emotional reaction, and that this would be predicted by difficult psychosocial circumstances at trial entry.

\section{Methods}

\section{Participants}

Nineteen children with DMD and their families were recruited into a phase II open-label, six-cohort dose escalation study of an i.v. administered antisense oligomer (AVI-4658) which restores the reading frame in amenable cases of DMD and may induce dystrophin restoration. All 19 took part in the psychosocial evaluation. The trial was conducted in two different children's hospitals and was approved by the relevant institutional ethics committees. The main results have been reported elsewhere (Cirak et al. 2011): this demonstrated the potential of AVI-4658 as a disease-modifying drug in the treatment of DMD, as it induced skipping of exon 51 in all children, followed by the appearance of dystrophin protein in those given the higher doses. Dystrophin-associated proteins were also restored while muscle inflammation was reduced; no drug-related serious adverse events were recorded.

\section{Procedure}

For the trial - which lasted up to 38 weeks - informed consent was obtained from families. Children were screened up to 12 weeks prior to the first dose of study drug; during the 12-week treatment period they received weekly drug infusions, provided urine and blood samples and underwent routine safety assessments. Participants had three additional safety evaluations at monthly intervals and a muscle biopsy (in two children under general anaesthesia). Safety evaluations were further conducted 2 weeks after the last dose of the study was administered.

The psychosocial evaluation involved psychosocial/ psychiatric interviews with parents and children at the trial screening phase conducted by psychiatrists/psychologist and complemented by questionnaires; parents also completed questionnaires at trial completion to assess the trial's emotional impact and any changes in psychosocial/psychiatric status.

\section{Measures}

At trial entry information was obtained about sociodemographic circumstances, previous and current child and parent psychiatric adjustment, as well as on the practical and emotional impact on parents and families of major past concerns about the child's illness (scored on an 11-point scale, with 10 representing the highest impact). Parent and child expectations from the trial were scored by the interviewer as 'realistic', 'possibly unrealistic' or 'definitely unrealistic' and in addition, parents rated on an 11-point scale the expected emotional impact of the trial on family members.

Serious concerns about the family's ability to cope with the trial procedures were regarded as exclusion criteria, but in the event no families were excluded on this basis.

\section{Family psychosocial and psychiatric risk assessment questionnaires}

To assess family psychosocial stressors and supports we used the Parental Stress and Supports Questionnaire (PSSQ; Bailey \& 
Garralda 1987). This is rated by parents in eight domains (occupation, finances, housing, social relationships with extended family and with friends, marriage, child with DMD and other children at home) and provides information about the degree of stress and support experienced at the time of assessment. Each domain is rated according to the degree of stress/support experienced on a 4-point scale ( 2 indicating 'marked' and 3 'extreme' levels of stress/support). A total score is derived by summing the individual items (maximum score of 24 for stress and support respectively).

Parents also completed the General Health Questionnaire (GHQ-28; Goldberg 1978) about their own mental health. This is a well-validated questionnaire assessing mental distress in adults; the threshold score of $\geq 5$ indicates high psychiatric risk.

\section{Child psychiatric risk assessment questionnaires}

Parents completed the Strengths and Difficulties Questionnaire (SDQ) to document psychiatric risk or emotional and behavioural problems experienced by the child in the previous 6 months. The SDQ provides one total problems and five subscale scores (emotional, conduct, hyperactivity, peer problems and prosocial behaviour) as well as an impact score. Validated cut-offs indicating risk for psychiatric disorder are available; a total score of $\geq 17$ indicates high risk for psychiatric disorder, a score of $\geq 5$ on the emotional scale indicates a risk for an emotional disorder and an impact score of $\geq 2$ is considered an indication of a clinical problem.

\section{Trial impact scores}

As main outcome measure, at trial completion parents were asked to indicate on an 11-point Likert scale the actual emotional impact from participating in the trial on the child and each parent ( 0 indicating none and 10 high impact). They also indicated the nature of the impact, i.e. whether positive, negative or neutral, and they completed the same psychosocial and psychiatric questionnaires as at trial entry to assess change in psychiatric status.

Data analysis included frequency counts, correlation and comparative data analysis. We used Wilcoxon signed-rank and Mann-Whitney tests and one-tailed Spearman correlations as appropriate.

\section{Results}

Both parents were interviewed in 12 (63\%) cases. Demographic details are given in Table 1 . The mean child age was 8.9 years
(SD 2.1) and most children were at the late ambulatory stage in the disease (they were able to walk with difficulty).

At trial entry psychosocial/psychiatric difficulty was present in a minority of families (Table 1). High current psychosocial stress on the PSSQ was reported by $15 \%$ and $11 \%$ of mothers were at risk of mental distress on the GHQ; a past history of family depressive disorder was noted by $16 \%$. High risk for current psychiatric disorder in the child on the SDQ was found in one child. Expectations about the trial were judged to be appropriate in all families and mean expected emotional impact scores were low.

During the trial psychological concerns were raised with regards to three children. One child was noted to be becoming anxious and upset about the trial and its possible side effects and was seen again by the child psychiatrist; the anxieties were allayed at interview and the child entered and completed the trial successfully. A second child had learning and behavioural difficulties and there was concern about his ability to co-operate with the trial infusions; this was discussed between the child psychiatrist, parents and trial staff, and a satisfactory procedure agreed. The third child was reluctant to have his final trial biopsy following earlier difficulties with venupuncture; he was re-assessed by the child psychiatrist and it was agreed not to proceed with the biopsy.

At the end of the trial 18 parents reported actual trial impact scores on the child, 17 on mothers and 13 on fathers (Fig. 1). The nature of the impact was reported for 14 children, 12 mothers and 10 fathers. Actual impact scores were higher than expected scores and correlations between them low for child impact ( $r=0.12 ; P=\mathrm{NS}$ ), but statistically significant for mother $(r=0.57 ; P=0.009)$ and father scores $(r=0.56 ; P=0.04)$.

There was generally good agreement between comments made by the parents and the nature and degree of impact they reported. Some inconsistency was noted in the scores of one mother who rated the impact on the child as 5 and neutral, but commented that he had been tired and worried about missing school; she rated the impact on herself as 8 and as neutral but described the trial as stressful and tiring for her.

\section{Emotional impact of the trial on the child}

Figure 1 shows that actual impact on the child was rated as positive in nearly half the cases and as neutral in over a quarter. Median impact scores for both positive and negative ratings were comparable ( 8 and 7 out of 10 respectively), while the median score for neutral ratings were lower (2/10). Positive comments included statements that children enjoyed the process and meeting people as well as other boys with DMD, and that they were proud to take part in the trial and felt some 
Table 1. Sample characteristics and psychosocial factors at trial entry $(n=19)$

\begin{tabular}{|c|c|c|c|c|}
\hline \multicolumn{5}{|l|}{ Demographic data } \\
\hline \multirow[t]{2}{*}{ Mean age } & \multicolumn{2}{|c|}{8.95 years (SD 2.1) } & & \\
\hline & \multicolumn{3}{|c|}{$n(\%)$} & \\
\hline \multicolumn{2}{|l|}{ Living with two biological parents } & \multicolumn{2}{|c|}{$12(63)$} & \\
\hline \multicolumn{2}{|l|}{ Father in external employment } & \multicolumn{2}{|c|}{$16(84)$} & \\
\hline \multicolumn{2}{|l|}{ Mother in external employment } & \multicolumn{2}{|c|}{$11(58)$} & \\
\hline \multicolumn{2}{|l|}{ White ethnicity $(n=18)$} & \multicolumn{2}{|c|}{$17(94)$} & \\
\hline \multicolumn{2}{|l|}{ Attending mainstream school } & \multicolumn{2}{|c|}{$13(68)$} & \\
\hline \multicolumn{2}{|l|}{ Below average educational performance $(n=18)$} & \multicolumn{2}{|c|}{$4(22)$} & \\
\hline \multirow[t]{2}{*}{ Family history of depression } & & \multicolumn{2}{|c|}{$3(16)$} & \\
\hline & & Mean (SD) & Median (quartile) & $n(\%)^{*}$ (at risk) \\
\hline \multirow[t]{2}{*}{ Impact on family of past major illness concerns } & Practical & $2.4(3.2)$ & $1(0,4)$ & $4(21)$ \\
\hline & Emotional & $2.8(3.7)$ & $1(0,5)$ & $5(26)$ \\
\hline \multirow[t]{2}{*}{ Expected emotional impact from trial } & On child & $2.1(2.1)$ & $1(0,4)$ & $4(21)$ \\
\hline & On mother & $3.2(2.8)$ & $4(1,4)$ & $3(16)$ \\
\hline \multicolumn{2}{|c|}{ Current psychosocial family stress total score (PSSQ) $(n=13)$} & $6.5(4.4)$ & $6(4,9)$ & $2(15)$ \\
\hline \multicolumn{2}{|c|}{ Current psychosocial family support total score (PSSQ) $(n=13)$} & $13.3(5.1)$ & $11.5(9,7.25)$ & \\
\hline \multicolumn{2}{|l|}{ Satisfaction with support for child's illness $(n=18)$} & $6.9(2.6)$ & $7(6,9)$ & $2(11)$ \\
\hline \multicolumn{2}{|l|}{ Current maternal distress (GHQ) $(n=17)$} & $1.4(3.3)$ & $0(0,0.5)$ & $2(11)$ \\
\hline \multicolumn{2}{|l|}{ Current paternal distress (GHQ) $(n=10)$} & $0.1(0.3)$ & $0(0,0)$ & 0 \\
\hline \multirow[t]{7}{*}{ Child SDQ (parent-rated) } & Total score & $8.8(4.7)$ & $10(5,12)$ & $1(5)$ \\
\hline & Impact score $(n=12)$ & $0.6(1.4)$ & $0(0,0.75)$ & $1(8)$ \\
\hline & Emotional sub-score & $1.8(1.5)$ & $1(1,3)$ & $1(5)$ \\
\hline & Peer relationship sub-score & $1.6(1.5)$ & $2(0,2)$ & $2(11)$ \\
\hline & Prosocial sub-score & $7.9(1.8)$ & $8(7,10)$ & $2(11)$ \\
\hline & Conduct sub-score & $1.7(1.5)$ & $1(0,3)$ & $3(16)$ \\
\hline & Hyperactivity sub-score & $3.7(2.3)$ & $4(2,6)$ & $2(11)$ \\
\hline
\end{tabular}

*The number of families at risk, i.e. scoring above/below mid-point on the PSSQ/impact/satisfaction scores, or above the cut-offs for psychiatric disorder on GHQ \& SDQ.

GHQ, General Health Questionnaire; PSSQ, Parental Stress and Supports Questionnaire; SDQ, Strengths and Difficulties Questionnaire.

good was being done. A parent noted that the child was a bit scared at first but was fine once the trial started.

Negative comments included the hospital being a bit scary for the child, stress regarding injections and blood samples, and realizing a deterioration in the condition as the trial went on, with depression that nothing positive had come out of it after the considerable effort made.

The comparison of child SDQ psychiatric questionnaire scores before and after the trial revealed few differences, median change scores being zero for parentally rated child SDQ total problems, conduct, hyperactivity, peer relationship and impact scores. Only the change in the child's SDQ emotional sub-scores (positive median change of 1 , or mean change of 0.56 ) indicated an increase in symptoms. Nevertheless, these changes were unrelated to the emotional impact scores.

\section{Emotional impact of the trial on parents}

Figure 1 shows that mothers reported the highest median actual impact scores but, as with the children, this was mainly rated as positive in nature. Comments reflected excitement at being part of the trial, an ability to do something for a condition currently without cure, seeing improvements in the child's walking, and also in everyday management. Negative impact was reported by two mothers (who also gave the highest negative impact scores for their children) and reflected the stress of spending 1 day a week on the trial, tiredness, confusion, and having to remain positive when the child found the process (such as injections) difficult.

Fathers generally reported the lowest impact scores of all three respondents per family, and their comments were very similar to the mothers'. For the rest of the analysis therefore only child and mothers' results are considered.

Comparisons of the maternal psychosocial stress and mental distress questionnaire scores before and after the trial revealed few differences. Median change scores were zero for maternal mental distress on the GHQ and median change in the total psychosocial stress score small $(-0.50)$. As with the children, there were not associations between maternal psychiatric change and trial impact scores. 
Expected and actual emotional trial impact median scores (10=max impact)

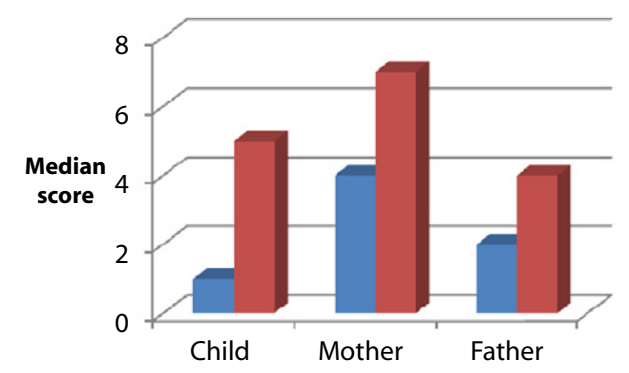

- Expected impact

= Actual impact

Nature of actual emotional impact from trial

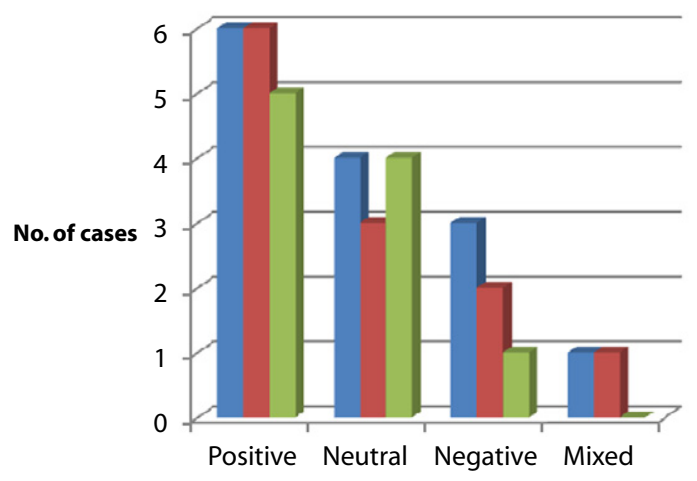

Median child actual emotional impact scores by type of impact

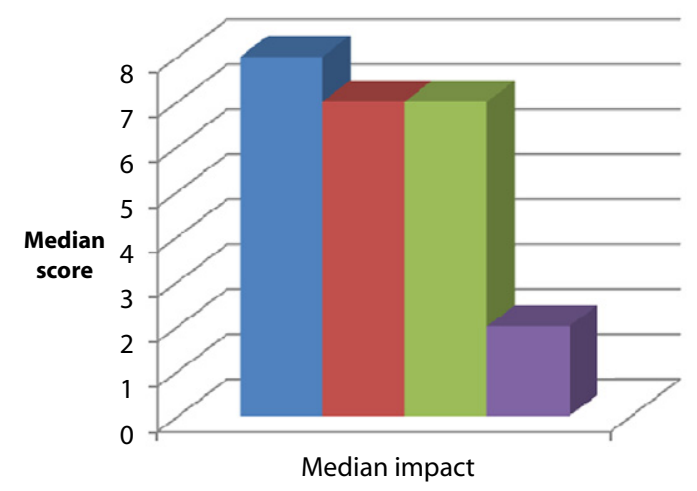

nositive

negative

aixed

n Neutral

Figure 1. Expected and actual trial emotional impact scores in children and parents.

Associations with actual negative trial impact on the child

We considered whether the negative impact reported for three of the children could have been predicted by any of the psychosocial factors measured at trial entry. However, the only statistical association was with the expected impact scores, which
Table 2. Psychosocial stressors in the three families reporting negative impact from the trial on the child

\begin{tabular}{llll}
\hline & Family $\mathbf{1}$ & Family $\mathbf{2}$ & Family $\mathbf{3}$ \\
\hline Trial negative emotional impact score & 9 & 7 & 4 \\
Practical impact from child illness in the & - & + & - \\
$\quad$ past & & & \\
Expected trial impact & + & + & - \\
PSSQ total stress & + & $+(-)^{*}$ & + \\
Maternal GHQ total & - & - & + \\
Parental marital stress & - & + & - \\
Child SDQ emotional score & + & - & - \\
Satisfaction with support with illness & - & ++ & - \\
Single-parent family & - & - & + \\
\hline
\end{tabular}

*Different ratings by mother/father.

tSecond lowest rating of whole group.

$+=$ highest/single adverse scores in the whole group.

GHQ, General Health Questionnaire; PSSQ, Parental Social Stress and Supports Questionnaire; SDQ, Strengths and Difficulties Questionnaire.

were significantly higher among these children [median $5(2,6)$ vs. $0.5(0,2.5)$; Mann-Whitney $P=0.031$; mean 4.3 (SD 2.1) vs. 1.2 (SD 1.8)].

None of the children who had received psychiatric assistance during the trial rated the impact negatively, nor was this explicitly linked by any of the parents to untoward physical effects of the trial over and above the comments made about injections/ cannulation.

Since this was an exploratory study and the statistical power to identify predictors of impact small, we examined whether there were any obvious risks shared by the three families reporting an actual negative impact on the children. Table 2 shows that these families had the highest total stress scores of all on the PSSQ at trial entry. In addition, individual families scored highly practical impact in the past from concerns about the child's illness, maternal mental distress and child emotional symptoms; they included the only single-parent family, another reporting a stressful parental situation and the family with the second lowest level of satisfaction with the amount of support received in managing their child's illness. But it was the combination of problems in various areas simultaneously that appeared relevant.

\section{Discussion}

In this dose-ranging exon-skipping trial in primary-school aged children with DMD, an emotional impact from the trial was reported by most families, more so than anticipated by them at trial entry. Impact was mostly rated as positive in nature and there were only minimal changes in family psychosocial and psychiatric status over the course of the trial. Nevertheless, a 
negative trial impact was reported for a minority of children and mothers. Preliminary exploration indicates that a combination of different types of psychosocial stressors may predict the development of negative emotional reactions and is worth exploring further.

It is important to note that families had volunteered for this study and a self-selection process is likely to have taken place, appropriately for a trial making considerable practical and emotional demands on families. Most were well adjusted psychosocially and psychiatrically at trial entry. The results might have been different if less psychosocially robust families had been sampled.

All the families expressed adequate overall knowledge and expectations about the trial, making therapeutic misconceptions an unlikely source of negative emotional impact (Kimmelman \& Palmour 2005; Henderson et al. 2006). This appears at variance with the report by Chappuy and colleagues (2010) in which $19 \%$ of parents signing consent forms for a trial in childhood leukaemia had not even realized that their child had been included in a research protocol, and half the parents could not explain the aim of the clinical trial nor the potential benefit of inclusion for their child. It is possible - following identification of distressing misconceptions in one family in a prior proofof-concept study (Garralda et al. 2011) - that the explanatory emphasis of our research team was greater. Alternatively, better understanding of the trial might be easier to achieve in families who already have a good deal of knowledge about a progressive disease such as in DMD or with small trial samples such as ours.

The fact that emotional impact from the trial was mostly rated as 'positive' or 'neutral' and that the trial did not affect the child or parents' psychiatric status indicates that in adequately selected families, and when good research support is provided, children and their parents may actually feel a benefit. This information should be helpful to ethics committees when considering clinical research with children (Angell et al. 2010). It is worth noting that the support available to the research units in the current study included psychiatric input, and it is interesting that none of the children who received psychiatric assistance during the trial rated its impact as negative (two scored it as positive and one as neutral).

However, a minority of families reported experiencing a negative impact citing distress over the physical procedures and disappointment about their child's deterioration as the cause. The exploratory nature of this study does not allow a statistical exploration of possible predictors of impact; nevertheless, there are indications that a combination of pre-trial risk factors with expectations of an emotional impact from the trial and high levels of psychosocial stress at the time of entering the study may be relevant. Psychosocial screening incorporating these risks can be easily included at the trial assessment stage. It would seem appropriate to further explore their relevance for the likelihood that children with progressive and other chronic disorders and their families experience a negative emotional impact from clinical trials.

\section{Key messages}

- In carefully selected families with low levels of psychosocial stress and mental distress, well prepared and supported by paediatric research units (including psychiatric input when thought to be required), genetic trials in progressive disorders such as DMD are most likely to have a positive or neutral impact and lead to little change in the psychiatric status of the child or parent.

- A negative impact is, however, reported by a minority of families, connected with disappointment and difficulty with the trial process.

- It would be helpful to further explore possible psychosocial predictors of negative emotional impacts from paediatric trials.

\section{Acknowledgements}

We thank the participating patients and their families, the charities Muscular Dystrophy Campaign, Action Duchenne and the Duchenne Family Support Group for participating in the UK MDEX consortium, which undertook this study. We thank Kanagasabai Ganeshaguru for study co-ordination in London, Maria Kinali for constructive discussion in early stages about the trial design and Geoff Bell for patient co-ordination in Newcastle upon Tyne. We are grateful for the support of the NIHR Biomedical Research Centre Funding Scheme and the Somers Clinical Research Facility at Great Ormond Street Hospital, UCL Institute of Child Health, and in particular thank Anna Massey, Katie Rees and Elizabeth Leach. This work was supported by the Newcastle NIHR Clinical Research Facility, in particular the study nurses Linda Smith and Dorothy Carman. We are very grateful for Mariacristina Scoto's help in the clinical care of the patients. We also acknowledge the collaborations of the North Star Clinical network, which contributed to the recruitment of participants via the clinical colleagues Adnan Manzur, Stephanie Robb, Helen Roper, Rosaline Quinlivan and Louise Hartley. The study was supported by the MRC Centre for Neuromuscular diseases at UCL and Newcastle including the MRC Neuromuscular Centre Biobank. F. M. is supported by 
Great Ormond Street Hospital Children's Charity. Newcastle University, UCL and Oxford are partners in TREAT-NMD (EC036825). The study was sponsored by AVI BioPharma (Bothell, WA, USA). We are grateful to Nicole Hickey for the statistical analysis.

\section{References}

Angell, E., Biggs, H., Gahleitner, F. \& Dixon-Woods, M. (2010) What do research ethics committees say about applications to conduct research involving children? Archives of Disease in Childhood, 95, 915-917.

Bailey, D. \& Garralda, M. E. (1987) The use of social stress and support interview in families with deviant children: methodological issues. Social Psychiatry, 22, 209-215.

Chappuy, H., Baruchel, A., Leverger, G., Oudot, C., Brethon, B., Haouy, S., Auvrignon, A., Davous, D., Doz, F. \& Treluyer, J. M. (2010) Parental comprehension and satisfaction in informed consent in paediatric clinical trials: a prospective study on childhood leukaemia. Archives of Disease in Childhood, 95, 800-804.

Cirak, S., Arechavala-Gomeza, V., Guglieri, M., Feng, L., Torelli, S., Anthony, K., Abbs, S., Garralda, M. E., Bourke, J., Wells, D. J.,
Dickson, G., Wood, M. J. A., Wilton, S. D., Straub, V., Kole, R., Shrewsbury, S. B., Sewry, C., Morgan, J., Bushby, K. \& Muntoni, M. (2011) Exon-skipping and dystrophin restoration in patients with Duchenne muscular dystrophy after systemic phosphorodiamidate morpholina oligomer treatment: an open-label, phase 2, dose-escalation study. The Lancet, 378, 595-605.

Garralda, M. E., Muntoni, F., Cunniff, A. \& Caneja, A. J. (2006) Knee-ankle-orthosis in children with Duchenne muscular dystrophy: user views and adjustment. European Journal of Paediatric Neurology, 10, 186-191.

Garralda, M. E., Kinali, M., Cirak, S., Guglieri, M., Straub, V. \& Muntoni, F. (2011) Emotional impact of a paediatric exon-skipping therapy trial. Developmental Medicine and Child Neurology, 12, 1157-1159.

Goldberg, D. (1978) Manual of the General Health Questionnaire. NFER Publishing Company, Windsor, UK.

Henderson, G. E., Easter, M. M., Zimmer, C., King, N. M. P., Davis, A. M., Rothschild, B. B., Churchill, L. R., Wilfond, B. S. \& Nelson, D. K. (2006) Therapeutic misconception in early phase gene transfer trials. Social Science and Medicine, 62, 239-253.

Kimmelman, J. \& Palmour, N. (2005) Therapeutic optimism in the consent forms of phase 1 gene transfer trials: an empirical analysis. Journal of Medical Ethics, 31, 209-214. 